\title{
Sonication fluid culture of antibiotic-loaded bone cement spacer has high accuracy to confirm eradication of infection before reimplantation of new prostheses
}

\author{
Qingyu Zhang ${ }^{1}$, Baocong Ding ${ }^{2}$, Jinglin $\mathrm{Wu}^{3}$, Jun Dong ${ }^{1}$ and Fanxiao Liu ${ }^{1 *}$ (D)
}

\begin{abstract}
Background: Sonication fluid culture of antibiotic-loaded bone cement spacer has been used to predict reinfection of two-stage revision, but its value remains disputable. This study aims to evaluate the association between the culture result of the sonicated spacer and the status of patients with periprosthetic joint infection receiving twostage revision.

Materials and methods: A comprehensive electronic literature search was performed through four databases including PubMed, Embase/Ovid, and EBSCO, and the Cochrane Library to retrieve studies in which sonication fluid culture of the antibiotic spacer was conducted before reimplantation. The pooled sensitivity, specificity, positive likelihood ratio (PLR), negative likelihood ratio (NLR), and diagnostic odds ratio (DOR) were calculated to assess the association between the culture result of sonicated spacer and prognosis of the two-stage revision.

Results: Eleven eligible studies comprising 603 artificial joints with PJI (134 suffering a clinical failure of two-stage revision) were included in the quantitative analysis. The pooled incidences of positive culture of sonicated spacer and intraoperative tissue were 0.14 (95\% confidence interval [Cl] 0.08-0.21) and 0.14 (95\% Cl 0.08-0.20), respectively. A positive culture of sonicated antibiotic-loaded bone cement spacer illustrated moderate sensitivity $(0.31,95 \% \mathrm{Cl}$ $0.13-0.58)$ but high specificity $(0.94,95 \% \mathrm{Cl} 0.86-0.98)$ for the diagnosis of therapeutic failure of two-stage revision; the pooled DOR was 7.67 ( $95 \% \mathrm{Cl}, 3.63-16.22)$. Meanwhile, the pooled sensitivity, specificity, and DOR of intraoperative tissue culture during the two-stage revision to predict therapeutic failure were $0.32(95 \% \mathrm{Cl}, 0.20-$ 0.47), 0.96 (95\% Cl, 0.92-0.98), and 10.62 (95\% Cl, 4.90-23.01), respectively.

Conclusions: Sonication fluid culture of antibiotic-loaded bone cement spacer revealed high accuracy for confirming eradication of infection before reimplantation of new prostheses and therefore could be used as a supplement for assessing therapeutic effect for PJl. However, both sonication fluid culture and intraoperative tissue culture from antibiotic-loaded bone cement spacer showed restricted yield for the prediction of a septic failure after the two-stage revision of PJI. Large-scale, prospective studies are still needed to testify current findings.
\end{abstract}

Keywords: Periprosthetic joint infection, Sonication culture, Spacer, Meta-analysis

\footnotetext{
* Correspondence: woshi631@126.com; liufanxiao@sdfmu.edu.cn

'Department of Orthopedics, Shandong Provincial Hospital affiliated to

Shandong First Medical University, No.324, Road Jing Wu Wei Qi, Jinan

250021, Shandong, China

Full list of author information is available at the end of the article
}

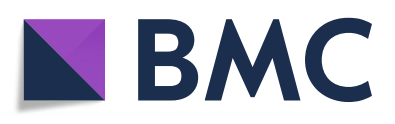

(- The Author(s). 2021 Open Access This article is licensed under a Creative Commons Attribution 4.0 International License, which permits use, sharing, adaptation, distribution and reproduction in any medium or format, as long as you give appropriate credit to the original author(s) and the source, provide a link to the Creative Commons licence, and indicate if changes were made. The images or other third party material in this article are included in the article's Creative Commons licence, unless indicated otherwise in a credit line to the material. If material is not included in the article's Creative Commons licence and your intended use is not permitted by statutory regulation or exceeds the permitted use, you will need to obtain permission directly from the copyright holder. To view a copy of this licence, visit http://creativecommons.org/licenses/by/4.0/. The Creative Commons Public Domain Dedication waiver (http://creativecommons.org/publicdomain/zero/1.0/) applies to the data made available in this article, unless otherwise stated in a credit line to the data. 


\section{Introduction}

Two-stage exchange arthroplasty is the most commonly recommended treatment protocol for chronic periprosthetic joint infection (PJI) of the knee and hip arthroplasty [1]. After removal of the infected implants and debridement of infected and necrotic tissue, an articulating or static bone cement (polymethyl methacrylate/ PMMA) spacer impregnated with antimicrobial agents is inserted to preserve the joint space and enable local antibiotic delivery; then, a systemic antibiotic therapy was administrated before a second prosthesis [2]. Although high success rate had been reported with an infection eradication rate above $80 \%$ for both total knee [3] and hip [4] arthroplasties, there are still risks of revision failure involving persistent or recurrent infection after reimplantation $[5,6]$. Presumably being multi-factorial, the formation of biofilm-forming pathogens (Fig. 1) on the bone cement spacer is likely the paramount cause for the infection to persist $[5,6]$. Once the embedded antibacterial molecules have exhausted, the antibioticloaded bone cement spacer (ACS) itself can serve as an optimal biomaterial surface to which bacteria can adhere, grow, and develop antibiotic resistance [7]. Meanwhile, relapse of PJI may be more frequent when drug-resistant organisms such as methicillin-resistance Staphylococcus aureus (MRSA) and methicillin-resistant Coagulase-negative Staphylococci (CoNS) were involved $[2,8]$.

Failure to eradicate PJI at the second-stage surgical procedure is associated with a decreased chance of favorable prognosis with a subsequent staged revision [9, 10] and may predispose patients with this catastrophic complication to the amputation, knee fusion, or Girdlestone procedure, leading to joint dysfunction and limited joint mobility $[5,11]$. Hence, an accurate method to predict persistent/recurrent infection after two-stage revision is highly desirable to avoid placing a new prosthesis in an infected environment. Unfortunately, conventional tools such as systemic inflammatory biomarkers, white blood cell count, the culture of synovial fluid, and intraoperative histopathology all revealed low ability to assess the infection eradication and treatment failure [8, 12, 13]. A recent study performed by our team revealed that

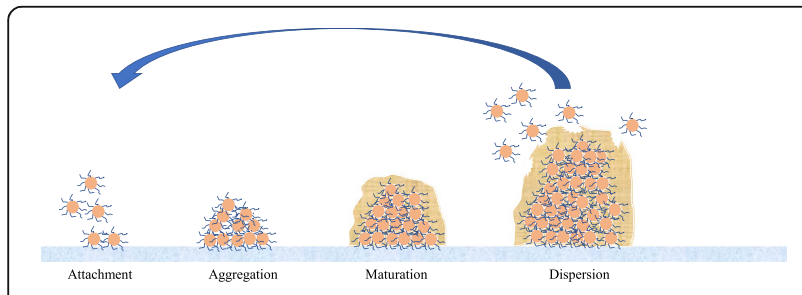

Fig. 1 Steps of microorganism biofilm formation and development plasma fibrinogen was an excellent biomarker for diagnosing PJI, comparable to serum CRP and ESR, while the diagnostic value of circulating $\mathrm{D}$-dimer was only moderate [14]. However, given the fact that the biofilm formed on the prosthesis surface may prevent microorganisms from being detected and eliminated, the culture of the sonicated prosthesis explanted during primary revision has been demonstrated to be more sensitive than the conventional periprosthetic tissue culture for diagnosing PJI $[15,16]$. Inspired by these findings, a series of small studies supported the application of sonicated culture of removed spacers before reimplantation and illustrated an association between a positive result, persistent or recurrent infection, and poor outcome [17-19]. However, these studies only included a small number of participants using qualitative, semi-quantitative, and quantitative sonication spacer fluid culture, making these results hard to be widely generalized. Moreover, data from other authors reported different experiences with the value of sonication fluid culture of bone cement spacer in comparison with conventional intraoperative tissue culture in the management of recurrent and persistent PJI [20, 21].

In order to further clarify this issue, a systematic literature search was conducted to retrieve studies evaluating the association between culture result of sonicated antibiotic-loaded bone cement spacers and occurrence of therapeutic failure after a two-stage revision procedure. Moreover, a meta-analysis was performed to reach more comprehensive results and provide high-quality evidence for the decision-making of clinicians.

\section{Materials and methods}

The methodological approach to evidence searching and data synthesis described in the current study and metaanalysis was in line with the standards of the Preferred Reporting Items for a Systematic Review and Metaanalysis of Diagnostic Test Accuracy Studies (PRISMADTA) [22]. No ethical approval or informed consent was required in this article because all data were retrieved from published literature. Study searching, eligibility identification, data extraction, and quality assessment were performed by two investigators independently. Any disagreement would have to be resolved through discussion, and the two researchers would have to reach a consensus.

\section{Search strategy}

Four electronic databases (PubMed, Embase/Ovid, and EBSCO, and the Cochrane Library) were searched for entries recorded from the time of database inception to March 15, 2021, using a combination of keywords and $\mathrm{MeSH}$ terms including "spacer" and "periprosthetic joint infection". No limitations were imposed on the journal 
and language of publication. Meanwhile, bibliographies of relevant articles were also hand-screened to retrieve any additional possible records.

\section{Inclusion criteria}

Studies included in systematic review need to meet all following criteria: (1) participants, patients receiving two-stage revision for PJI; (2) intervention, sonication fluid culture of removed spacers; (3) control, the diagnosis of PJI was confirmed by the Musculoskeletal Infection Society (MSIS), American Academy of Orthopaedic Surgeons (AAOS), or Infectious Diseases Society of America (IDSA) guidelines; (4) outcome, adequate data could be extracted to calculate the positive incidence of sonication fluid culture or diagnostic accuracy of this test for persistent infection; and (5) study design, diagnostic accuracy study.

Exclusion criteria were (1) case reports/series, metaanalyses, editorials, commentaries, expert opinion, and narrative reviews, and (2) studies in which PJI could not be diagnosed with golden standards.

The titles and abstracts were independently assessed in an unblinded standardized manner for eligibility. The final decision regarding eligibility was based on the fullarticle scrutinizing. If more than one study provided overlapping data, only the most comprehensive or latest one was included.

\section{Data extraction and quality assessment}

Requisite data extracted and recorded to standardized excel files included surname of the first author, year and region of publication, study inclusion interval, study design, demographic characteristics of enrolled participants (e.g., sex, age), criteria for the diagnosis of PJI, the method to conduct culture of sonication spacer, number of positive/negative case of sonication fluid culture, and prognosis of two-stage revision. Clinical failure was defined as recurrence/existence of PJI after two-stage revision.

The methodological quality of included studies was appraised according to the QUADAS (Quality Assessment of Diagnostic Accuracy Studies)-2 tool which contains four key domains, namely, patient selection, index test, reference standard, and flow and timing [23]. The risk of bias in each domain and concerns about applicability were assessed in the first three domains. Questions answered with "yes" indicated a low risk of bias/ concern, "no" a high risk of bias/concern, and "unclear" that relevant information was not provided [23].

\section{Statistical analyses}

The pooled positive incidence of sonication fluid culture of the explanted spacer was computed by generating the proportion of the yield (true-positive) and associated
95\% confidence intervals (CI) using a random effects model. For the diagnostic modalities, true-positive (TP), false-positive (FP), true-negative (TN), and false-negative (FN) results were extracted from the two-by-two contingency table to calculate the pooled sensitivity, specificity, positive likelihood ratio (PLR), negative likelihood ratio (NLR), diagnostic odds ratio (DOR), and summary receiver operating characteristic (sROC). Heterogeneity among the included studies was assessed using the $I^{2}$ statistic. An $I^{2}$ value of $0-50 \%$ implied non-significant heterogeneity, and values of $>50 \%$ indicated substantial heterogeneity. Publication bias was performed using Deeks' funnel plot asymmetry test. All meta-analyses were conducted using the STATA (V. 12.0, StataCorp, College Station, TX). The value of a two-sided $p<$ 0.05 was considered statistically significant in all statistical tests.

\section{Results}

\section{Selection process}

A total of 117 potentially eligible articles were retrieved after the original search of three electronic databases, and 21 additional articles were retrieved from the references of relevant articles (meta-analyses, systematic reviews, letters, editorials, and guidelines). We excluded 62 ineligible articles by screening the titles and abstracts. After reading the full text of the remaining articles, 11 studies [17, 19-21, 24-30] involving a total of 583 participants were included in the statistical analysis, all being published in English. The study selection process is presented as a flow chart in Fig. 2.

\section{Study characteristics}

The detailed characteristics of the enrolled studies are summarized in Table 1. These studies were published from 2012 to 2019 with the sample sizes ranged from 13 to 157 . A total of 583 participants involving 603 artificial joints were included, among which therapeutic failure (persistent or recurrent infection) occurred in 134 joints. The included studies investigated artificial hip, knee, shoulder, and elbow joints. Three studies $[17,20,26]$ are conducted in the USA, and eight $[19,21,24,26-30]$ from Europe. Ten studies [17, 19, 20, 24-30] provided the results of sonication fluid culture from spacers, and one [21] used PCR analysis of sonication fluid from spacers. Nine studies $[17,19,24-30]$ also provided the culture results of intraoperative tissue of bone cement spacers. Five [17, 19, 21, 28, 29] studies used a prospective design and the others $[20,24-27,30]$ a retrospective cohort design. The QUASDAS-2 scores of qualities ranged from 9 to 11 with a mean of 10.27. Treatment failure was defined as existence of infection after twostage revision for PJI. 


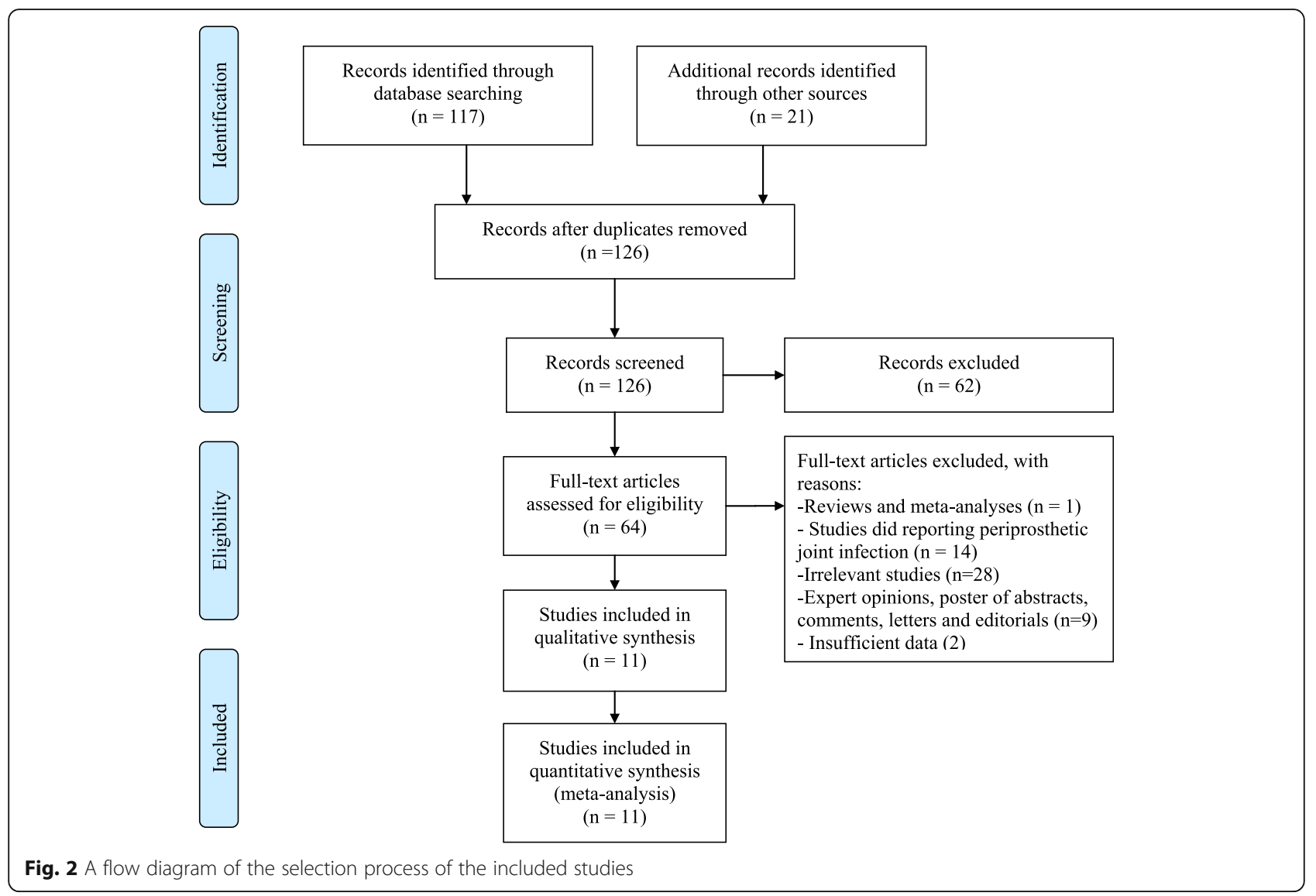

\section{Qualitative analysis}

\section{Incidence of a positive result}

The overall incidence of a positive result of sonication fluid culture of bone cement spacers as generated from 10 datasets $[17,19,20,24-30]$ was $14 \%$ (95\% CI $12-$ $21 \%)$ at the second surgical stage during a two-stage exchange procedure (Fig. 3a). For the intraoperative tissue culture of bone cement spacers, the overall incidence as generated from 9 datasets $[17,19,24-30]$ was $14 \%$ (95\% CI 8-20\%), demonstrating a similarly positive result with sonication fluid culture (Fig. 3b).

\section{Diagnostic accuracy of sonicated spacer culture}

Results assessing the value of sonication fluid culture of antibiotic-loaded bone cement spacer for diagnosing revision failure as generated from the 10 datasets [17, 19$21,24,26-30]$ included in the present meta-analysis showed a sensitivity of 0.31 ( $95 \% \mathrm{CI}, 0.13$ to 0.58$)$, a specificity of 0.94 (95\% CI, 0.86 to 0.98 ), a PLR of 5.37 (95\% CI, 2.83 to 10.20), an NLR of 0.73 (95\% CI, 0.53 to 0.99 ), a DOR of 7.38 (95\% CI, 3.33 to 16.38), and an AUC of 0.83 (95\% CI, 0.79 to 0.86 ) (Figs. 4, 5a, and 6a). The threshold effect was found in the provided data (Spearman correlation coefficient $=0.753 ; p$ value $=0.007$ )
Deeks' funnel plot asymmetry test revealed no publication bias $(p$ value $=0.97)($ Fig. $7 \mathrm{a})$.

\section{Diagnostic accuracy of intraoperative tissue culture}

Performance assessing the diagnostic value of tissue culture of antibiotic-loaded bone cement spacer for PJI as generated from the 9 datasets $[17,19,21,24,26-30]$ included in the present meta-analysis showed a sensitivity of 0.32 (95\% CI, 0.20 to 0.47 ), a specificity of 0.96 (95\% CI, 0.92 to 0.98 ), a PLR of 7.58 (95\% CI, 3.86 to 14.85), an NLR of 0.71 (95\% CI, 0.59 to 0.87 ), a DOR of 10.62 (95\% CI, 4.90 to 23.01), and an AUC of 0.84 (95\% CI, 0.80 to 0.87 ) (Figs. $5 \mathrm{~b}$ and $6 \mathrm{~b}, 8$ ). The threshold effect was not found in the provided lesion-based data (Spearman correlation coefficient $=0.345 ; p$ value $=0.328)$. Deeks' funnel plot asymmetry test revealed no publication bias $(p$ value $=0.45)($ Fig. $7 \mathrm{~b})$.

\section{Discussion}

The two-stage exchange is the standardized protocol in the treatment of PJI as it not only provides local antimicrobial delivery to the joint but also preserves the normal anatomy of the joint space, facilitating delayed reimplantation. However, there are still cases of revision failure where the cement spacer itself may act as a 


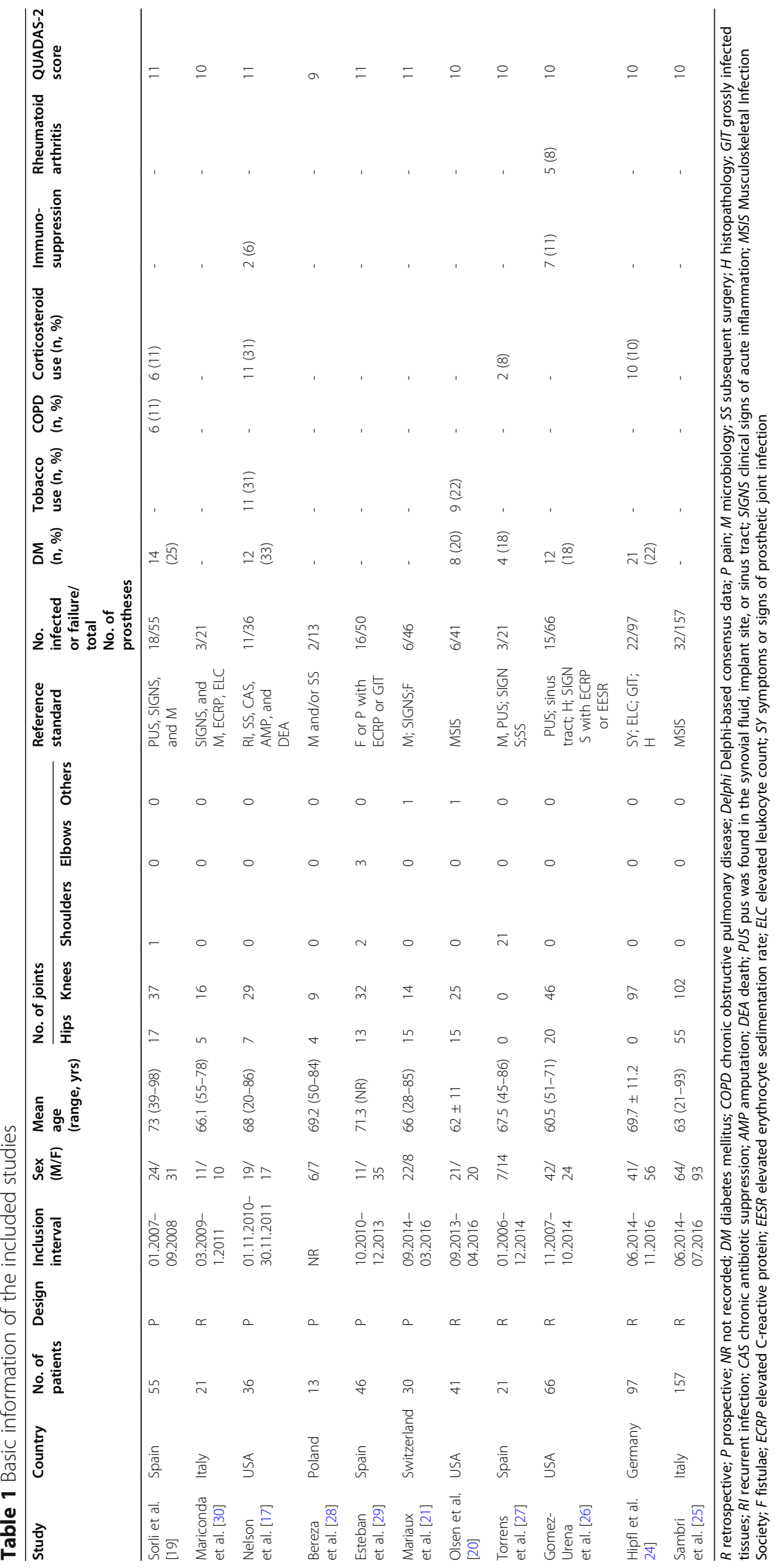


a

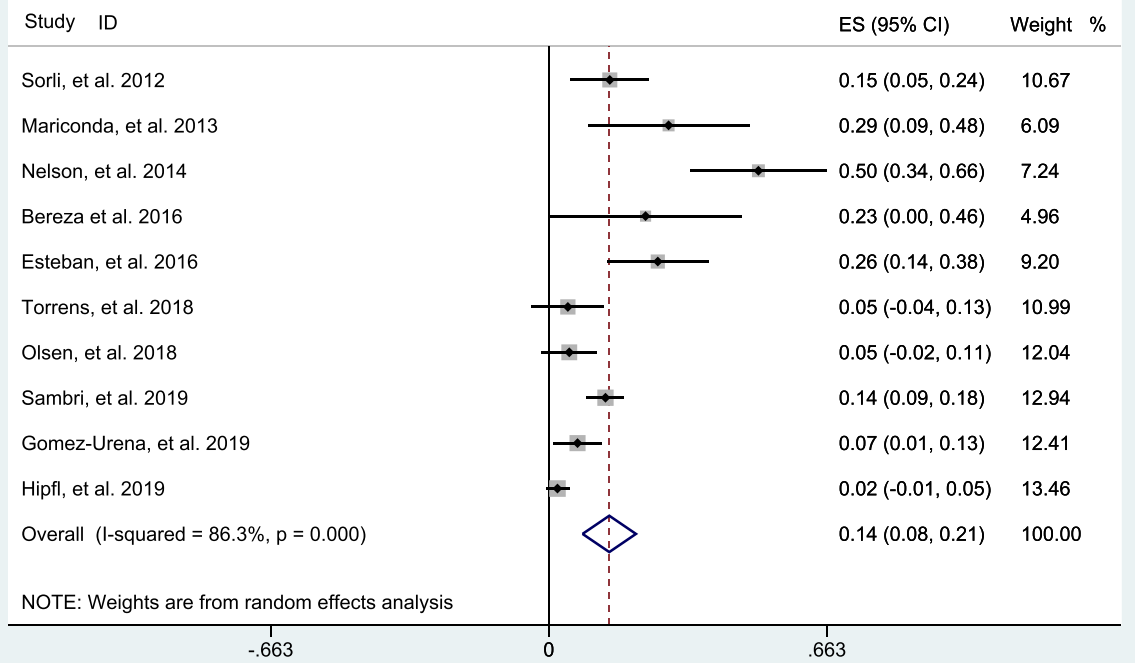

b

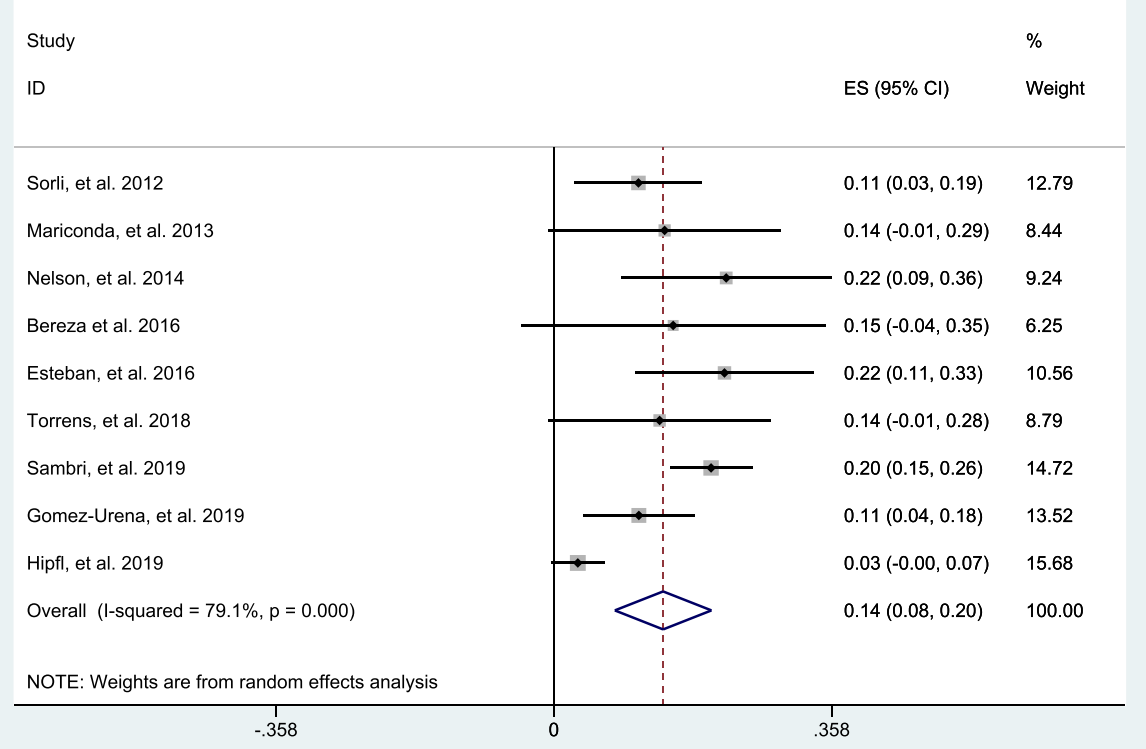

Fig. 3 a The overall incidence of a positive result of sonication fluid culture of bone cement spacers in patients receiving two-stage exchange revision. $\mathbf{b}$ The overall incidence of a positive result of the intraoperative tissue culture of bone cement spacers in patients receiving two-stage exchange revision

biomaterial surface that predisposes the survival and proliferation of microorganisms [7]. Moreover, the early identification of the pathogens involved in septic failure after reimplantation may help in giving pathogenspecific suppressive antibiotic therapy as soon as possible after surgery [31]. The prediction of a persistent/recurrent infection is mainly achieved by the culture of intraoperative tissue or synovial fluid before replacement of new implants, and various synovial or serological biomarkers, but a definitive diagnosis of this catastrophic complication remains a tough issue [32,33]. The current study demonstrated that the sonication fluid culture of explanted spacers revealed a similar incidence $(14 \%)$ of a positive result to that of intraoperative tissue culture. These two methods showed comparable diagnostic sensitivity (31\% and 32\%, respectively) and specificity ( $94 \%$ 


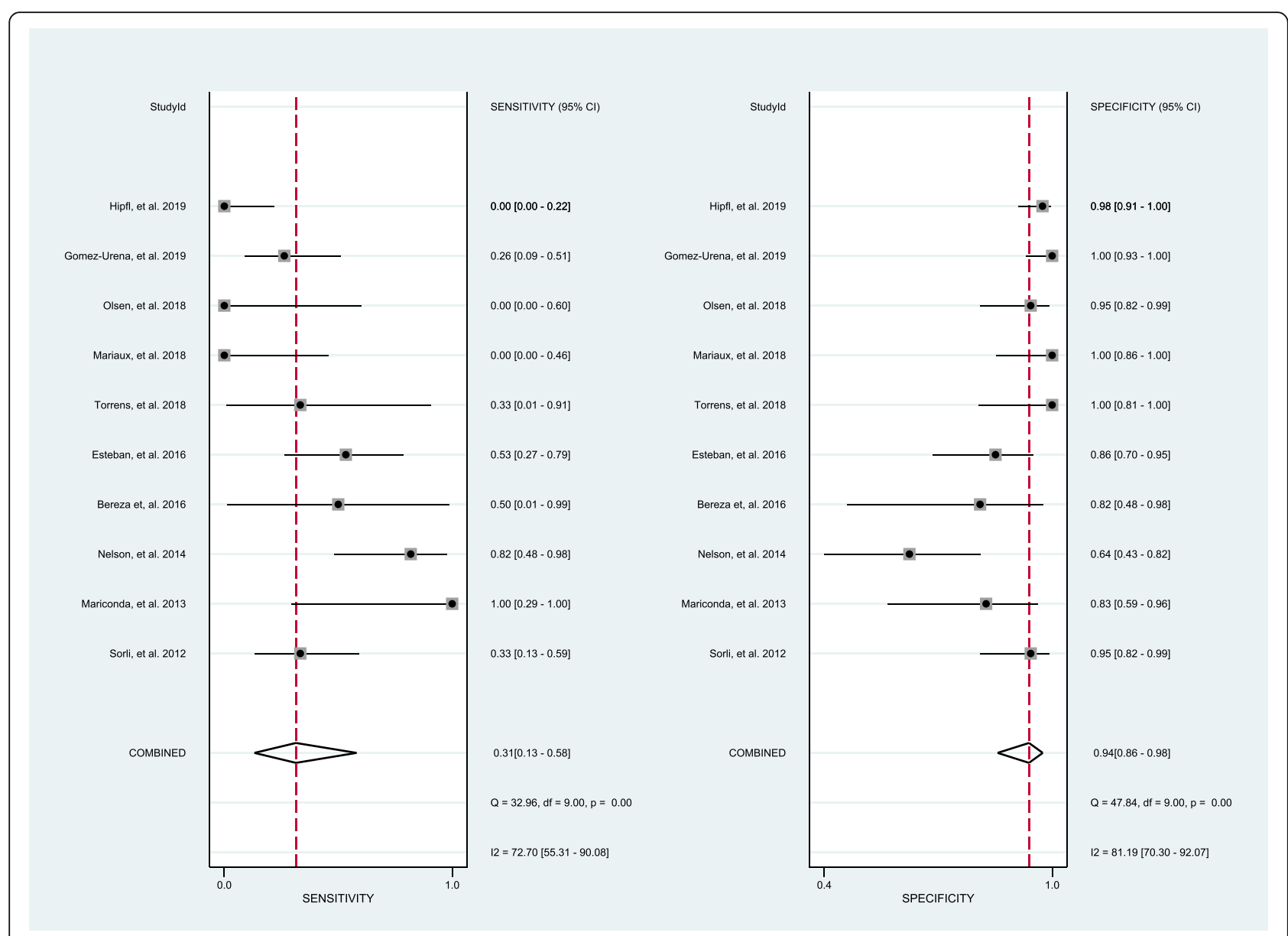

Fig. 4 The pooled sensitivity and specificity of sonication fluid culture of bone cement spacers in patients receiving two-stage exchange revision

and $96 \%$, respectively) for the prediction of septic failure of two-stage revision.

Septic failure of a two-stage revision of PJI can be attributed to either insufficient debridement of the previous infection (persistent infection) or a newly onset infection with a different pathogen [34]. These two groups of patients pose distinct predisposing factors and infection causes [8]. A previous study involving $37 \mathrm{pa}-$ tients with PJI caused by oxacillin-resistant staphylococci reported that septic failure of two-stage revision occurred in $24.32 \%$ (9/37) cases, despite all patients having negative periprosthetic tissue cultures at the time of reimplantation of new prostheses, from which $44.44 \%$ (4/ 9) of patients had a recurrent infection with the same organism and $55.56 \%(5 / 9)$ revealed reinfection with a different organism [8]. In Hipfl et al.' study [24], 22 patients receiving two-stage revisions of total knee arthroplasty suffered clinical failure, and only 3 reinfections are caused by the same organism. In Olsen et al.'s study [20], two cases with positive sonication fluid culture of spacers revealed distinct results with the initial infection.
Although isolation of the same pathogen from periprosthetic tissue culture and ACS culture served as the golden diagnostic criteria of persistent PJI, since genotypic analysis on bacterial strains was not conducted in included studies, these cases considered as relapses might be new infections not detected during the second surgery or that occurred after implantation. Several recent studies indicated that septic failure after two-stage revisions was mainly due to the new infection, rather than the persistent infection $[13,35]$. The ACS, as a foreign body, could not only allow persistence of preexisting infection but also facilitate adherence of new organism. It had to be emphasized that the complicated and prolonged procedures of two-stage exchange were indeed associated with a higher risk of introducing new pathogens in comparison with the primary procedure [36]. Other possible explanations for these newly identified pathogens were that these patients had a polymicrobial infection, and the additional species were not revealed at the first-stage surgery or only represent a contamination of the sample [24]. These reasons could 
a

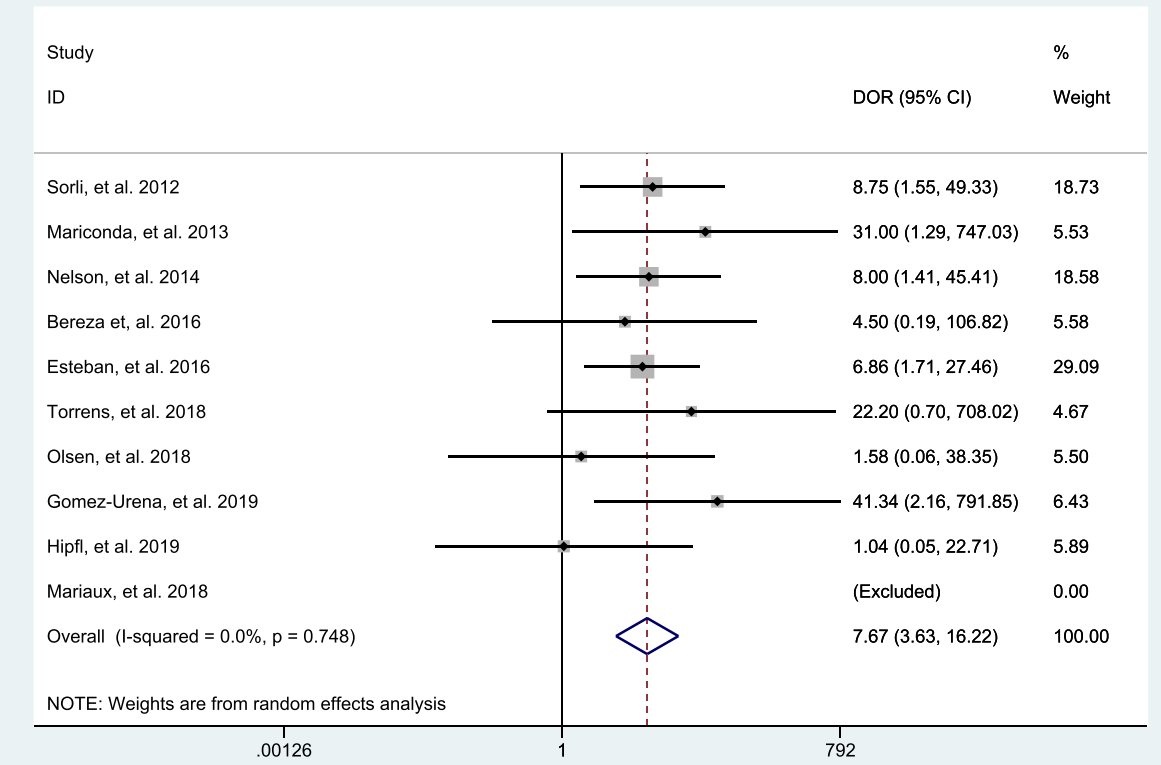

b

Study
ID

Fig. 5 The diagnostic odds ratio of sonication fluid culture (a) or intraoperative tissue culture (b) of bone cement spacers in patients receiving two-stage exchange revision

partially explain why microbiological tests before reimplantation of new artificial joints, such as intraoperative tissue culture and sonication fluid culture of the explanted prostheses and inflammatory markers, lack sensitivity to detect subsequent infections during the second-stage surgery [35]. Therefore, further studies are needed to seek host factors, genetic predisposition, and misconduct of medical staff as causes of septic failure of staged revision of PJI.
In our study, although a significant association between results of sonication fluid culture of the antibiotic-loaded spacer and aseptic failure was identified $(\mathrm{DOR}=7.38$; 95\% CI, 3.33 to 16.38), positive results of culture were only identified in less than one-third of patients with septic failures. Meanwhile, there were discordant results between periprosthetic tissue culture and sonication fluid culture of spacers. In Sambri et al.'s study [25], concordant results between two culture 

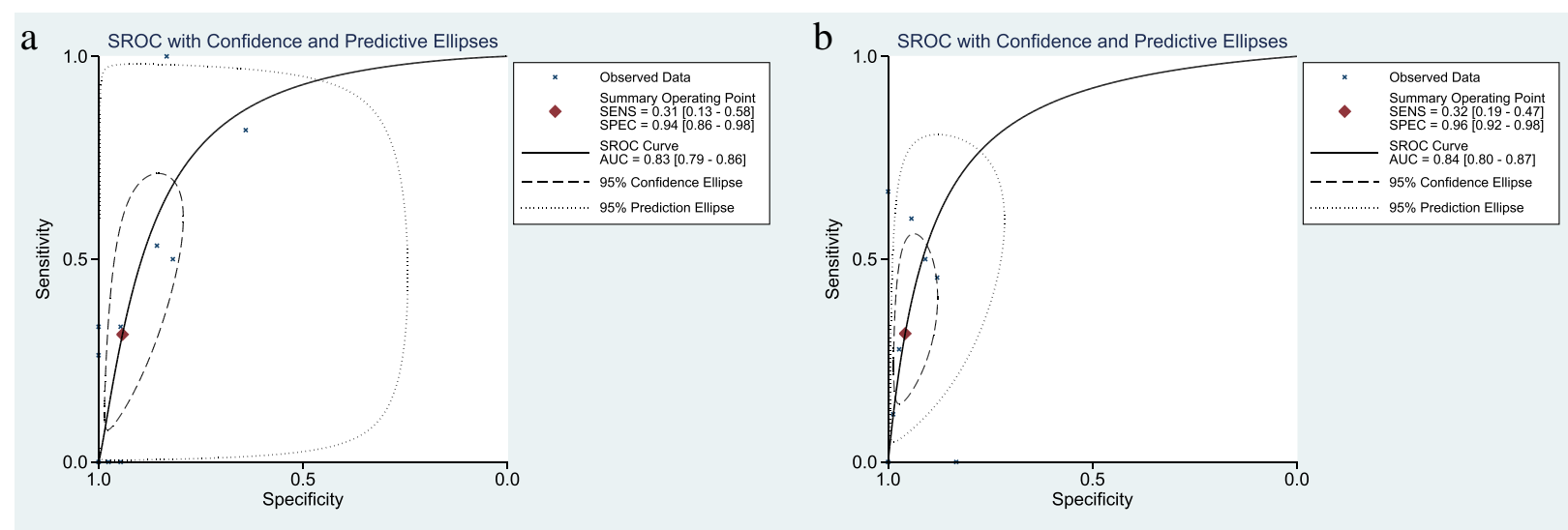

Fig. 6 The systemic receiver operating curve of sonication fluid culture (a) or intraoperative tissue culture (b) of bone cement spacers in patients receiving two-stage exchange revision

methods occurred in 186 (83.8\%) out of 222 cases. Among 31 cases with persistent infection and discordant results of two culture methods, 23 were only identified according to the periprosthetic tissue cultures of spacers and 8 only from cultures performed on sonication fluids of spacers. In addition to a new infection, the great majority of false-negative cases can be explained by the fact that the spacer would release antimicrobials out of the cement and result in inhibition of bacterial growth in sonication fluid culture [21, 37]. Mariaux et al. reported an antimicrobial concentration at or above minimal inhibitory concentration (MIC) of common PJI microorganisms in sonication fluid [21]. Meanwhile, preoperative antibiotic treatment can also affect the sonication fluid culture of bone cement spacer. Trampuz et al. reported that the sensitivities of periprosthetic tissue and sonication fluid cultures for diagnosing periprosthetic joint infection were $60.8 \%$ and $78.5 \%$, respectively in patients without antimicrobial therapy more than 14 days before surgery, and declined to $45.0 \%$ and $75.0 \%$ in those with an antibiotic course within 14 days before surgery [33]. Various antibiotic-free period before the secondstage surgical procedure may also influence the sensitivity of sonication fluid culture of bone cement spacer for the diagnosis of septic failure of the two-stage exchange protocol for PJI.

Albeit the sensitivity of ACS culture is limited, the utmost caution must be exercised when pathogens are isolated from sonication fluid of the cement spacer, particularly a highly resistant microorganism or same bacteria that caused the initial PJI. Long-term elution of antibiotics from bone cement spacer may lead to drug resistance in bacterial strains [38]. Before reimplantation of the prosthesis during a two-stage exchange protocol, a

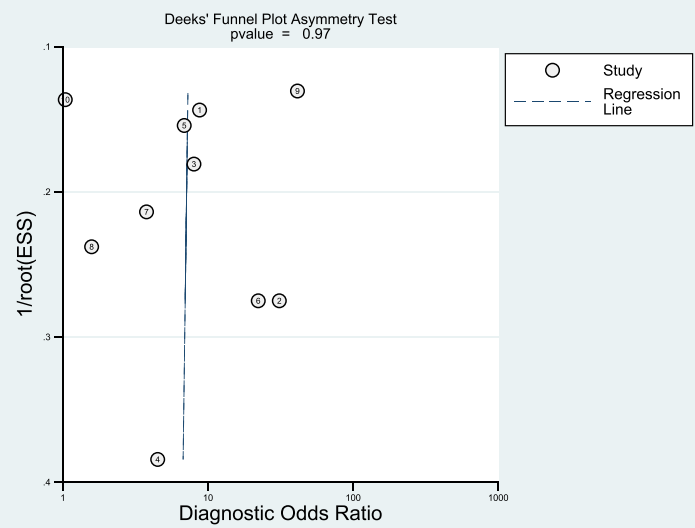

b

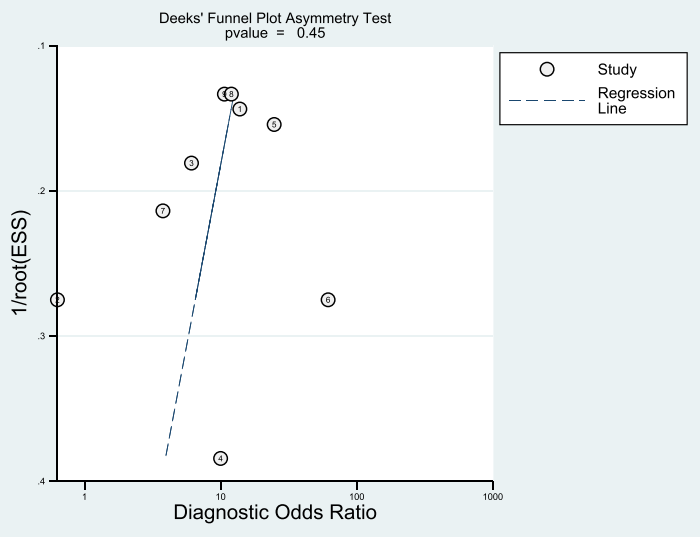

Fig. 7 Graphical display of the results of Deek's test for publication bias of sonication fluid culture (a) and intraoperative tissue culture (b) 


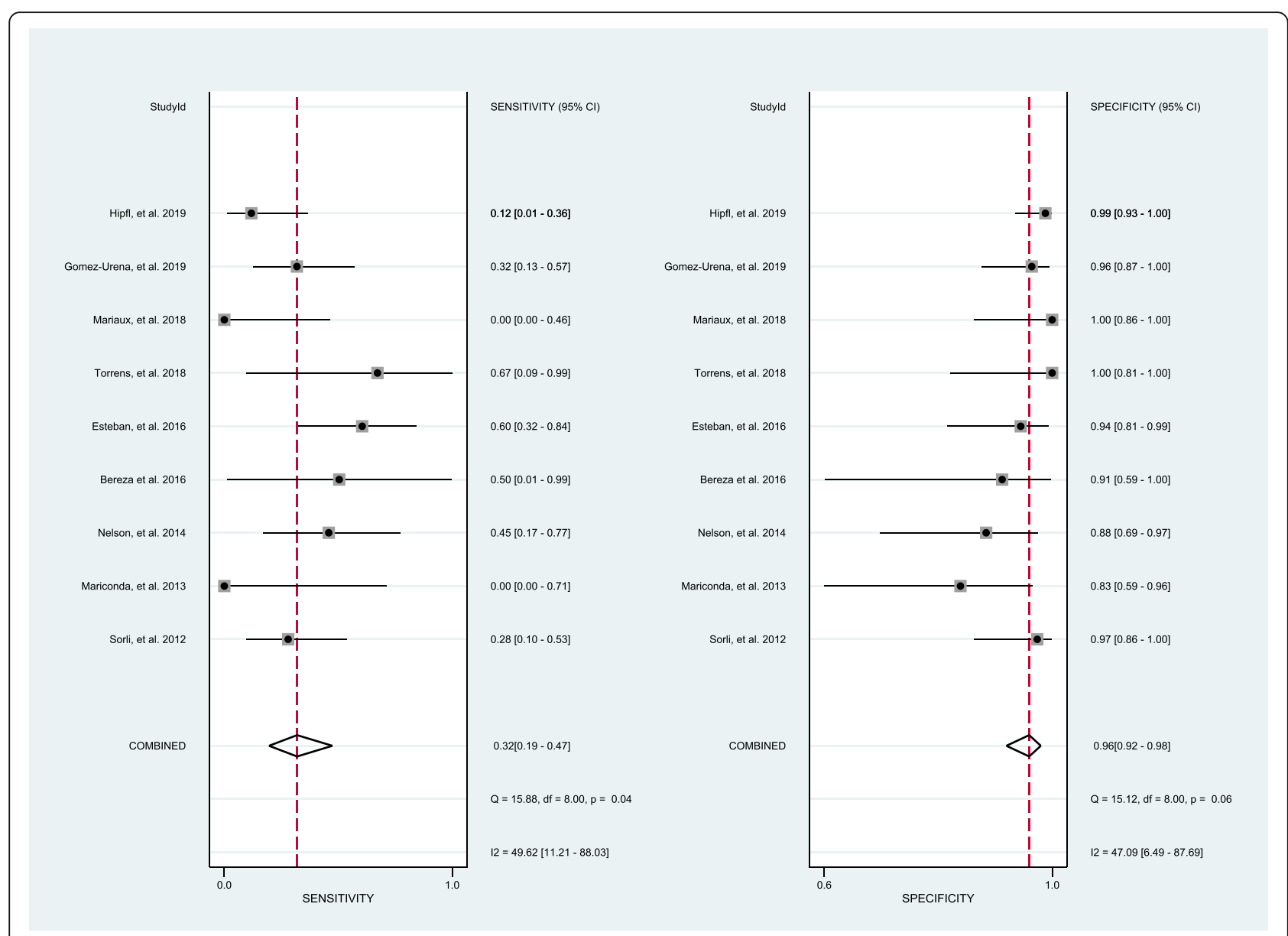

Fig. 8 The pooled sensitivity and specificity of intraoperative tissue culture of bone cement spacers in patients receiving two-stage exchange revision

a sonication fluid culture of the bone-cement spacer should be conducted to provide more information for the screening of infection. Meanwhile, different perspectives exist concerning the therapeutic regimen of different versus identical pathogens when comparing culture results of sonication fluid with primary PJI. In fact, for a patient with positive sonication fluid culture of bone cement spacer, an applicable algorithm should be established on host characteristics, organism species/ virulence, timing, and other variables.

There are limitations that should be considered with regard to the interpretation of current findings. The first is the lack of a standardized reference test for the diagnosis of septic failure of two-stage infection. Another minor limitation is that during the merging of diagnostic data, subgroup analyses on the basis of important information such as amount of culture fluid and duration of follow-up were not conducted due to the limited number of studies. Only 11 studies were included in this meta-analysis, and most $(6 / 11)$ of them were retrospective in nature. Third, the sample size of these studies was quite small, and hence, this meta-analysis might be subject to variability and inadequacy in data collection. Lastly, yet importantly, evidence of heterogeneity existed throughout the included studies, which was a significant limitation of the current investigation. Therefore, we emphasized the pooled incidence, and DOR, which is a global measure encompassing other diagnostic parameters as the main outcome of interest to reflect the performance of sonication fluid culture of bone cement spacer.

\section{Conclusions}

In conclusion, sonication fluid culture of antibiotic-loaded bone cement spacer revealed high accuracy for confirming eradication of infection before reimplantation of new prostheses and therefore could be used as a supplement for assessing therapeutic effect for PJI. However, both sonication fluid culture and intraoperative tissue culture from antibiotic-loaded bone cement spacer showed restricted yield for the prediction of a septic failure after the twostage revision of PJI. Large-scale, prospective studies are still needed to testify current findings. 


\section{Abbreviations}

AAOS: American Academy of Orthopaedic Surgeons; ACS: Antibiotic-loaded bone cement spacer; Cl: Confidence intervals; CoNS: Coagulase-negative Staphylococci; DOR: Diagnostic odds ratio; FN: False negative; FP: False positive; IDSA: Infectious Diseases Society of America; MIC: Minimal inhibitory concentration; MRSA: Methicillin-resistance Staphylococcus aureus:

MSIS: Musculoskeletal Infection Society; NLR: Negative likelihood ratio PJl: Periprosthetic joint infection; PLR: Positive likelihood ratio; PMMA: Polymethyl methacrylate; PRISMA-DTA: Preferred Reporting Items for a Systematic Review and Meta-analysis of Diagnostic Test Accuracy Studies; QUADAS: Quality assessment of diagnostic accuracy studies; sROC: Summary receiver operating characteristic; TN: True negative; TP: True positive

\section{Acknowledgements}

Not applicable.

\section{Authors' contributions}

LFX and ZQY contributed to the idea of this study. LFX and ZQY searched literatures and screened them independently. LFX, ZQY, and WJL screened data and made tables. LFX, ZQY, and WJL played an important role in analyzing the outcomes. LFX, ZQY, and WJL conducted the data analyses and made graphs. LFX, ZQY, DBC, and WJL wrote the first draft and polished and approved the final version. The authors read and approved the final manuscript.

\section{Funding}

The design, collection, analysis, and interpretation of the data in the study were financially supported by the China Scholarship Council (CSC) (NO. 201808080126) and the Young Taishan Scholars Program of Shandong Province (NO. tsqn201909183), the Academic promotion programme of Shandong First Medical University (NO. 2020RC008), and the Natural Science Foundation of Shandong Province (NO. ZR201911090016). The authors, their immediate families, and any research foundations with which they are affiliated have not received any financial payments or other benefits from any commercial entity related to the subject of this article.

\section{Availability of data and materials}

All data analyzed during this study are included in this published article.

\section{Declarations}

\section{Ethics approval and consent to participate}

Not applicable.

\section{Consent for publication}

All participants signed informed consent forms for publication.

\section{Competing interests}

The authors declare that they have no competing interests.

\section{Author details}

'Department of Orthopedics, Shandong Provincial Hospital affiliated to Shandong First Medical University, No.324, Road Jing Wu Wei Qi, Jinan 250021, Shandong, China. ${ }^{2}$ Rehabilitation Department, Shandong University of Traditional Chinese Medicine Affiliated Hospital, No.16369, Road Jing Shi, Jinan 250014, Shandong, China. ${ }^{3}$ Basic Course Department, Weihai Vocational College, New Sci-Tech Park of Beihai, Weihai 264200, Shandong, China.

Received: 28 April 2021 Accepted: 2 June 2021

Published online: 13 June 2021

\section{References}

1. Kunutsor SK, Whitehouse MR, Lenguerrand E, et al. Re-infection outcomes following one- and two-stage surgical revision of infected knee prosthesis: a systematic review and meta-analysis. Plos One. 2016;11:e151537.

2. Kurd MF, Ghanem E, Steinbrecher J, Parvizi J. Two-stage exchange knee arthroplasty: does resistance of the infecting organism influence the outcome? Clin Orthop Relat Res. 2010;468(8):2060-6. https://doi.org/10.1 007/s11999-010-1296-6.
3. Jamsen E, Stogiannidis I, Malmivaara A, et al. Outcome of prosthesis exchange for infected knee arthroplasty: the effect of treatment approach. Acta Orthop. 2009;80(1):67-77. https://doi.org/10.1080/17453670902805064.

4. Senthi S, Munro JT, Pitto RP. Infection in total hip replacement: metaanalysis. Int Orthop. 2011;35(2):253-60. https://doi.org/10.1007/s00264-01 0-1144-z.

5. Berend KR, Lombardi AJ, Morris MJ, et al. Two-stage treatment of hip periprosthetic joint infection is associated with a high rate of infection control but high mortality. Clin Orthop Relat Res. 2013;471(2):510-8. https:// doi.org/10.1007/s11999-012-2595-x.

6. Cochran AR, Ong KL, Lau E, Mont MA, Malkani AL. Risk of reinfection after treatment of infected total knee arthroplasty. J Arthroplasty. 2016;31(9):15661. https://doi.org/10.1016/j.arth.2016.03.028.

7. Neut D, van de Belt H, Stokroos I, et al. Biomaterial-associated infection of gentamicin-loaded PMMA beads in orthopaedic revision surgery. J Antimicrob Chemother. 2001;47(6):885-91. https://doi.org/10.1093/jac/47.6.885.

8. Mittal Y, Fehring TK, Hanssen A, et al. Two-stage reimplantation for periprosthetic knee infection involving resistant organisms. J Bone Joint Surg Am. 2007;89(6):1227-31. https://doi.org/10.2106/00004623-20070600000010.

9. Kalra KP, Lin KK, Bozic KJ, Ries MD. Repeat 2-stage revision for recurrent infection of total hip arthroplasty. J Arthroplasty. 2010;25(6):880-4. https:// doi.org/10.1016/j.arth.2009.12.010

10. Stammers J, Kahane S, Ranawat V, Miles J, Pollock R, Carrington RWJ, et al. Outcomes of infected revision knee arthroplasty managed by two-stage revision in a tertiary referral centre. Knee. 2015;22(1):56-62. https://doi.org/1 0.1016/j.knee.2014.10.005.

11. Rodriguez-Merchan EC. Knee fusion or above-the-knee amputation after failed two-stage reimplantation total knee arthroplasty. Arch Bone Jt Surg. 2015:3(4):241-3.

12. Kusuma SK, Ward J, Jacofsky M, Sporer SM, Della Valle CJ. What is the role of serological testing between stages of two-stage reconstruction of the infected prosthetic knee? Clin Orthop Relat Res. 2011;469(4):1002-8. https:// doi.org/10.1007/s11999-010-1619-7.

13. George J, Kwiecien G, Klika AK, Ramanathan D, Bauer TW, Barsoum WK, et al. Are frozen sections and MSIS criteria reliable at the time of reimplantation of two-stage revision arthroplasty? Clin Orthop Relat Res. 2016;474(7):161926. https://doi.org/10.1007/s11999-015-4673-3.

14. Zhang Q, Dong J, Zhou D, Liu F Circulating D-dimer versus fibrinogen in the diagnosis of peri-prosthetic joint infection: a meta-analysis. Surg Infect (Larchmt). 2021;22(2):200-210, 2, DOI: https://doi.org/10.1089/sur.2019.298.

15. Liu K, Fu J, Yu B, et al. Meta-analysis of sonication prosthetic fluid PCR for diagnosing periprosthetic joint infection. Plos One. 2018;13:e196418.

16. Liu H, Zhang Y, Li L, Zou HC. The application of sonication in diagnosis of periprosthetic joint infection. Eur J Clin Microbiol Infect Dis. 2017;36(1):1-9. https://doi.org/10.1007/s10096-016-2778-6.

17. Nelson $\mathrm{CL}$, Jones RB, Wingert NC, Foltzer M, Bowen TR. Sonication of antibiotic spacers predicts failure during two-stage revision for prosthetic knee and hip infections. Clin Orthop Relat Res. 2014;472(7):2208-14. https:// doi.org/10.1007/s11999-014-3571-4.

18. Portillo ME, Salvado M, Alier A, et al. Advantages of sonication fluid culture for the diagnosis of prosthetic joint infection. J Infect. 2014;69(1):35-41. https://doi.org/10.1016/j.jinf.2014.03.002

19. Sorli L, Puig L, Torres-Claramunt $R$, et al. The relationship between microbiology results in the second of a two-stage exchange procedure using cement spacers and the outcome after revision total joint replacement for infection: the use of sonication to aid bacteriological analysis. J Bone Joint Surg Br. 2012;94:249-53.

20. Olsen AS, Wilson A, O'Malley MJ, Urish KL, Klatt BA. Are sonication cultures of antibiotic cement spacers useful during second-stage reimplantation surgery for prosthetic joint infection? Clin Orthop Relat Res. 2018;476(10): 1986-92. https://doi.org/10.1007/s11999.0000000000000257.

21. Mariaux S, Furustrand TU, Borens $O$. Diagnosis of persistent infection in prosthetic two-stage exchange: evaluation of the effect of sonication on antibiotic release from bone cement spacers. J Bone Jt Infect. 2018;3(1):3742. https://doi.org/10.7150/jbji.23668.

22. McGrath TA, Moher D, McInnes M, et al. Steps toward more complete reporting of systematic reviews of diagnostic test accuracy: Preferred Reporting Items for Systematic Reviews and Meta-Analyses of Diagnostic Test Accuracy (PRISMA-DTA). Syst Rev. 2019;8(1):166. https://doi.org/10.1186/ s13643-019-1090-9. 
23. Whiting PF, Rutjes AW, Westwood ME, Mallett S, Deeks JJ, Reitsma JB, et al. QUADAS-2: a revised tool for the quality assessment of diagnostic accuracy studies. Ann Intern Med. 2011;155(8):529-36. https://doi.org/10.7326/0003-4 819-155-8-201110180-00009.

24. Hipfl C, Winkler T, Janz V, Perka C, Müller M. Management of chronically infected total knee arthroplasty with severe bone loss using static spacers with intramedullary rods. J Arthroplasty. 2019;34(7):1462-9. https://doi.org/1 0.1016/j.arth.2019.03.053.

25. Sambri A, Maso A, Storni E, Donati ME, Pederzoli A, Dallari D, et al. Is sonication of antibiotic-loaded cement spacers useful in two-stage revision of prosthetic joint infection? J Microbiol Methods. 2019;156:81-4. https://doi. org/10.1016/j.mimet.2018.12.006.

26. Gomez-Urena E, Sierra RJ, Greenwood-Quiantance KE, et al. Sonication culture of antimicrobial agent-containing cement spacers removed during staged revisions for arthroplasty infection. J Clin Microbiol. 2019:57(2): e01483-18.

27. Torrens C, Santana F, Puig L, Sorli L, Alier A. Results of cement spacer sonication in the second stage of two-stage treatment of shoulder arthroplasty infection. J Orthop Surg Res. 2018;13(1):58. https://doi.org/10.11 86/s13018-018-0763-8.

28. Bereza P, Ekiel A, Augusciak-Duma A, et al. Comparison of cultures and 165 rRNA sequencing for identification of bacteria in two-stage revision arthroplasties: preliminary report. BMC Musculoskelet Disord. 2016;17(1):138. https://doi.org/10.1186/s12891-016-0991-1.

29. Esteban J, Gadea I, Perez-Jorge C, et al. Diagnosis of spacer-associated infection using quantitative cultures from sonicated antibiotics-loaded spacers: implications for the clinical outcome. Eur J Clin Microbiol Infect Dis. 2016;35(2):207-13. https://doi.org/10.1007/s10096-015-2531-6.

30. Mariconda M, Ascione T, Balato G, Rotondo R, Smeraglia F, Costa GG, et al. Sonication of antibiotic-loaded cement spacers in a two-stage revision protocol for infected joint arthroplasty. BMC Musculoskelet Disord. 2013; 14(1):193. https://doi.org/10.1186/1471-2474-14-193.

31. Siqueira MB, Saleh A, Klika AK, et al. Chronic suppression of periprosthetic joint infections with oral antibiotics increases infection-free survivorship. J Bone Joint Surg Am. 2015;97(15):1220-32. https://doi.org/10.2106/JBJS.N. 00999.

32. Berbari EF, Marculescu C, Sia I, Lahr BD, Hanssen AD, Steckelberg JM, et al. Culture-negative prosthetic joint infection. Clin Infect Dis. 2007;45(9):1113-9. https://doi.org/10.1086/522184

33. Trampuz A, Piper KE, Jacobson MJ, Hanssen AD, Unni KK, Osmon DR, et al. Sonication of removed hip and knee prostheses for diagnosis of infection. N Engl J Med. 2007;357(7):654-63. https://doi.org/10.1056/NEJMoa061588.

34. Tirumala V, Smith E, Box H, van den Kieboom J, Klemt C, Kwon YM. Outcome of debridement, antibiotics, and implant retention with modular component exchange in acute culture-negative periprosthetic joint infections. J Arthroplasty. 2020;36(3):1087-93. https://doi.org/10.1016/..arth.2 020.08.065.

35. Zmistowski B, Tetreault MW, Alijanipour P, Chen AF, Della Valle CJ, Parvizi J. Recurrent periprosthetic joint infection: persistent or new infection? J Arthroplasty. 2013;28(9):1486-9. https://doi.org/10.1016/j.arth.2013.02.021.

36. Rakow A, Perka C, Trampuz A, Renz N. Origin and characteristics of haematogenous periprosthetic joint infection. Clin Microbiol Infect. 2019; 25(7):845-50. https://doi.org/10.1016/j.cmi.2018.10.010.

37. Kummer A, Tafin UF. Borens O Effect of sonication on the elution of antibiotics from polymethyl methacrylate (PMMA). J Bone Jt Infect. 2017 2(4):208-12. https://doi.org/10.7150/jbji.22443.

38. Hsieh PH, Chang YH, Chen SH, Ueng SWN, Shih CH. High concentration and bioactivity of vancomycin and aztreonam eluted from Simplex cement spacers in two-stage revision of infected hip implants: a study of 46 patients at an average follow-up of 107 days. J Orthop Res. 2006;24(8):161521. https://doi.org/10.1002/jor.20214.

\section{Publisher's Note}

Springer Nature remains neutral with regard to jurisdictional claims in published maps and institutional affiliations. 